\title{
FDG PET/CT of a tyrosinemia child with metastatic hepatocellular carcinoma
}

\begin{abstract}
7 years old girl with known genetic disorder tyrosinemia type 1, recently diagnosed with hepatocellular carcinoma (HCC) and portal vein thrombosis. She was referred to our center to do FDG PET/CT study as a pre liver-transplant assessment which was her last therapeutic option. The aim of the PET/CT study was to exclude the neoplastic nature of the portal vein thrombus and any extra-hepatic malignancy (metastasis) which are a contraindication for liver transplant procedures. The study revealed aggressive infiltrating nature of such hepatic malignancy associated with vascular infiltration of the portal vein and metastatic pulmonary nodules. These results are a contraindication to the transplant procedure leaving no therapeutic options for this child. We found no illustrations in the literature of a previous PET/CT study with such findings in a tyrosinemia child making this case special.
\end{abstract}

Volume 8 Issue 6 - 2017

\author{
Emad Hamid,' Yehia Omar² \\ 'Department of Radiology, Ainshams University, Egypt \\ 2Department of PET/CT, Misr Radiology Center, Egypt
}

Correspondence: Efstathios Kamperis, Radiation Oncology Department, Papageorgiou General Hospital, Thessaloniki, Greece, Tel 302313323417 ,

Email omar.y@misradiologycenter.com

Received: June II, 2017 | Published: October 27, 2017

Keywords: Tyrosinemia; Hepatocellular carcinoma; PET/CT; Portal vein thrombosis

Abbreviations: HCC, hepatocellular carcinoma; FDG, fluorodeoxyglucose; SUV, standardized uptake value

\section{Case presentation}

A 7 years old girl was diagnosed with advanced stage of tyrosinemia type 1 at the age of 4 years. She started nitisinone therapy and dietary restriction to decrease the effects of the toxic metabolites. Recently, the patient's general condition deteriorated rapidly with marked rising in the alpha feto-protein level. Ultrasound examination revealed enlarged liver with marked cirrhotic features, extensive nodularity and masses occupying most of the liver parenchyma associated with portal vein thrombosis. Poorly differentiated hepatocellular carcinoma was then pathologically proven (highly pleomorphic tumor cells with giant multinucleated cells and bizarre nuclei with no apparent resemblance to hepatocytes). The only therapeutic option for this patient at this stage was liver transplantation, so she was referred for PET/CT examination to exclude any extra-hepatic malignancy or tumoral portal vein thrombosis. (which are contraindications to transplantation).

The PET/CT exam revealed the unexpected extensive nature of the disease which were a contraindication to the transplant procedure as follows;

\section{A. Malignant findings:}

i. Extensively cirrhotic liver with multiple heterogeneous large cirrhotic nodules and hypermetabolic malignant infiltration affecting most of the hepatic parenchyma achieving 15.5 SUVmax (Figures 1-4)

ii. The portal vein is markedly distended by hypermetabolic neoplastic thrombus occupying its whole course and both main branches. (Red arrow; Figures 2B,3B,4A,4B).

iii. Bilateral hypermetabolic metastatic pulmonary nodules. (Yellow arrow; Figure 6).

B. Non-malignant findings;

i. Bilateral enlarged kidneys showing marked cortical hold up of contrast (cortical nephrogram) with delayed contrast excretion (Figure 5) ii. Splenomegaly

iii. Marked ascites.

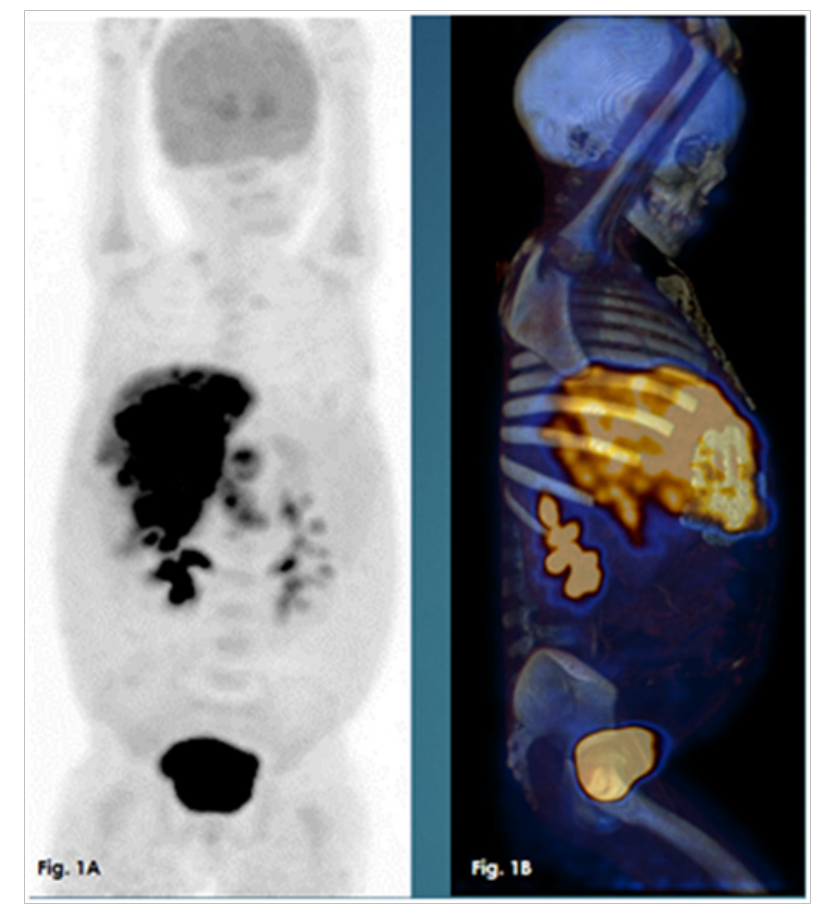

Figure I A whole body MIP PET images, IB: whole body MIP 3D PETCT images. Both showing hypermetabolic extensive liver infiltration.

Reviewing the literature revealed no previous illustrations of a PET/CT exam of tyrosinemia child with such findings of metastatic HCC, which makes this case very special.

\section{Discussion}

Tyrosinemia type 1 (also known as hepato-renal tyrosinemia) is a genetic disorder caused by a defect in the enzyme responsible for degradation of tyrosine, namely fumarylacetoacetase. As a result, multiple toxic metabolites are formed causing severe disruption of intracellular metabolism of the liver and kidney. These accumulated 
toxic metabolites cause increased hepatocyte apoptosis and oxidative stress, leading to chronic liver disease, cirrhosis and eventually predispose to hepatocellular carcinoma. The leading causes of death from tyrosinemia are fulminant liver failure, metastatic hepatocellular carcinoma, and porphyria-like neurologic crisis. ${ }^{1,2}$

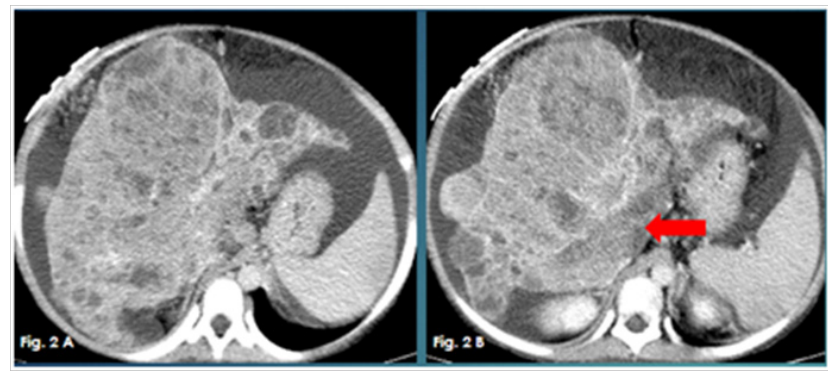

Figure 2A \& 2B Axial contrast enhanced CT images showing markedly cirrhotic liver studded with innumerable hypodense nodules and enhancing masses. Thrombosed portal vein (red arrow).

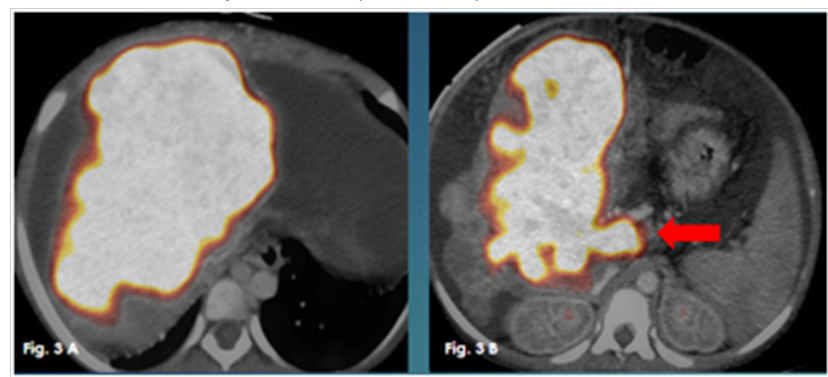

Figure 3 Axial fused PET/CT images showing the diffuse hypermetabolic malignant liver infiltration and the neoplastic nature of the portal vein thromboses (red arrow).

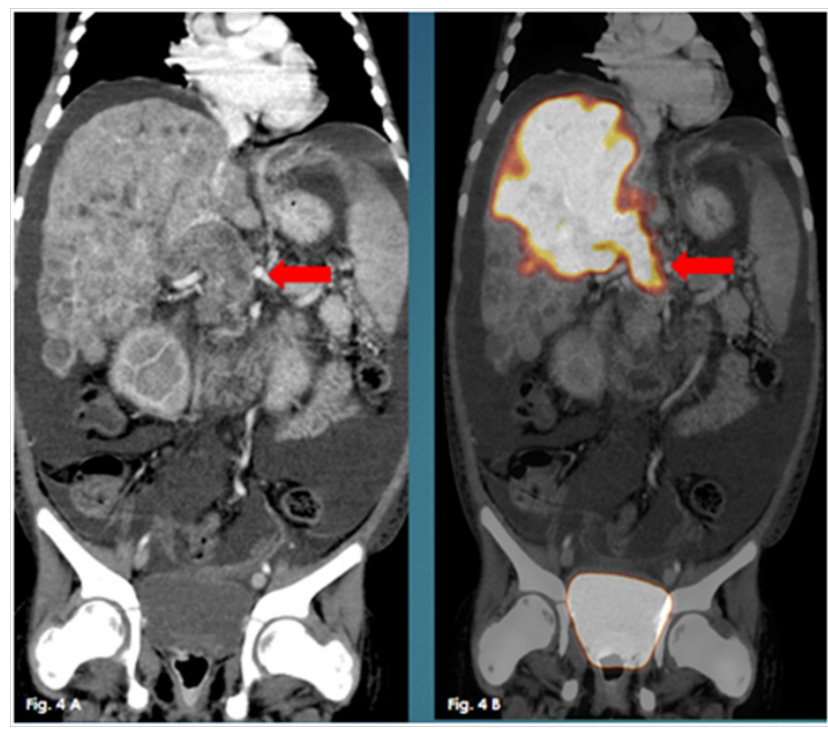

Figure 4 A Coronal enhanced CT, 4B: Coronal fused PET/CT. They show the multiple hypodense hypermetabolic hepatic focal lesions with neoplastic thrombosed portal vein.

Tyrosinemia type I, is the most severe form of this disorder and is characterized by signs and symptoms that begin in the first few months of life. Affected infants fail to grow at the expected rate due to poor food tolerance leading to diarrhea and vomiting. Some affected children have repeated neurologic crises that consist of changes in mental state, peripheral neuropathy, abdominal pain, and respiratory failure. Untreated, children with tyrosinemia type I often do not survive past the age of $10^{3}$. Tyrosinemia type I, has a birth incidence of approximately 1 in 100,000 in most areas but is more common in some regions, notably in Quebec, Canada. ${ }^{4}$

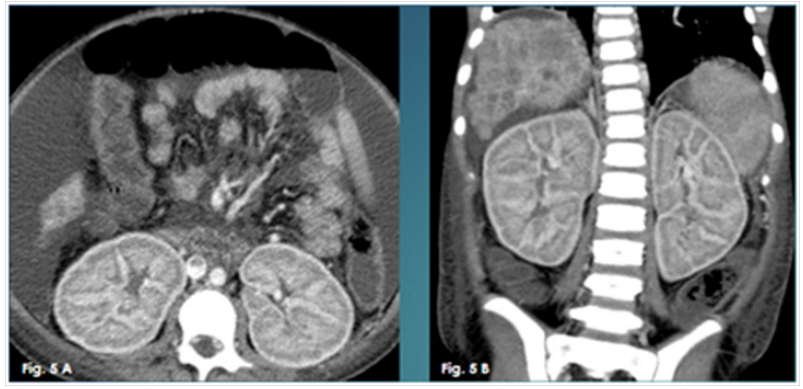

Figure 5 A Axial enhanced CT image, 5B: Coronal enhanced CT image. They both show markedly enlarged edematous kidneys with persistent cortical nephrogram.

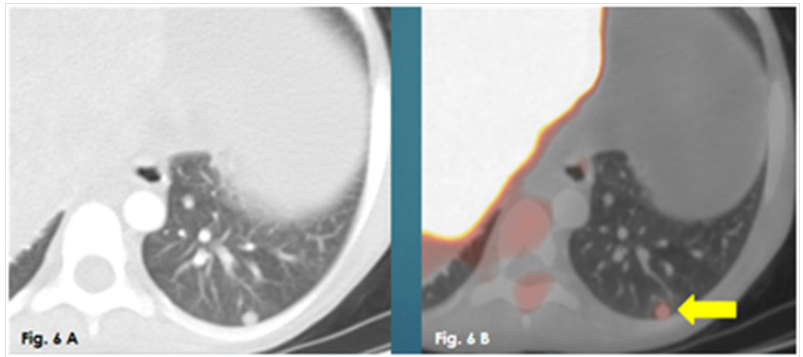

Figure 6 A Axial CT chest lung window, 6B:Axial fused PET/CT. They show increased metabolic activity in a left lower lung lobe metastatic pulmonary nodule.

Management approaches over the past few decades have resulted in a sharp decline in morbidity and mortality associated with this disease. Since Lindstedt first published his experience using nitisinone in 5 patients with Tyrosinemia in 1992, its incorporation into the standard treatment has resulted in improved effects on the long term outcome with improved metabolic control. ${ }^{5}$ In addition, newborn screening is a common practice in most Western countries allowing early identification and treatment of affected individuals as early as the first month of life. In the large Quebec experience, infants beginning treatment with nitisinone in the first month of life had no detectable liver lesions after more than 5 years of follow-up and no need for liver transplantation. The combined effects of nitisinone use and newborn screening have resulted in a significant reduction in the need for liver transplantation noted over the past decade. ${ }^{6}$

Our case was not fortunate enough to be discovered early to receive nitisinone therapy before hepatic and renal parenchymal damage and development of hepatocellular carcinoma. Management of hepatocellular carcinoma according to the Barcelona-Clinic Liver Cancer Group diagnostic and treatment strategy, states that surgical resection is considered the first treatment option for early stage patients. It is reserved for patients with solitary tumors without portal hypertension and normal bilirubin. If these conditions are not met, patients are considered for liver transplantation (cadaveric or live donation) or percutaneous ablation if at an early stage (solitary $<$ or $=5 \mathrm{~cm}$ or up to 3 nodules $<$ or $=3 \mathrm{~cm}$ ). These patients will reach a 5 -year survival between 50 and $75 \%$. If patients are diagnosed at an intermediate stage and are still asymptomatic and have preserved liver function, they may benefit from chemoembolization. Their 3-year survival will exceed $50 \%$. There is no effective treatment for patients with advanced disease and should receive only palliative treatment to avoid unnecessary suffering. 
In our case the patient had extensive liver infiltration with vascular extension and pulmonary metastatic nodules, which leaves the patient no therapeutic options and only palliative therapy.

\section{Conclusion}

Newborn screening for tyrosinemia should be routinely advocated as early therapy within the first month of life leads to significant reduction in liver disease, related cancers and mortality of these children.

\section{Conflicts of interest}

There is no conflict of interest.

\section{Acknowledgements}

None.

\section{Funding}

None.

\section{References}

1. Chakrapani A, Gissen P, McKiernan P. Inborn Metabolic Diseases Disorders of Tyrosine Metabolism, Chapter 18, Springer. 2012:275-276.
2. Fernández-Lainez C, Ibarra-González I, Belmont-Martínez L, et al. Tyrosinemia type I: clinical and biochemical analysis of patients in Mexico. Ann Hepatol. 2014;13(2): 265-272.

3. de Laet C, Dionisi-Vici C, Leonard JV, et al. Recommendations for the management of tyrosinaemia type 1. Orphanet Journal of Rare Diseases. 2013;8:8.

4. De Braekeleer M, Larochelle J. Genetic epidemiology of hereditary tyrosinemia in Quebec and in Saguenay-Lac-St-Jean. Am J Hum Genet. $1990 ; 47: 302-307$

5. Lindstedt S, Holme E, Lock EA, et al. Treatment of hereditary tyrosinaemia type I by inhibition of 4-hydroxyphenylpyruvate dioxygenase. Lancet. 1992;340:813-817.

6. Larochelle J, Alvarez F, Bussières JF, et al. Effect of nitisinone (NTBC) treatment on the clinical course of hepatorenal tyrosinemia in Québec. Mol Genet Metab. 2012;107:49-54.

7. Llovet JM, Fuster J, Bruix J. The Barcelona approach: Diagnosis, staging, and treatment of hepatocellular carcinoma. Liver Transpl. 2004;10: S115-S120. 